\title{
Local Information Based Algorithms for Packet Transport in Complex Networks
}

\author{
Bernard Kujawski ${ }^{1}$, G.J. Rodgers ${ }^{1}$, and Bosiljka Tadić ${ }^{2}$ \\ 1 Department of Mathematical Sciences, Brunel University, Uxbridge, \\ Middlesex UB8 3PH, UK \\ \{bernard.kujawski, g.j.rodgers\}@brunel.ac.uk \\ 2 Department for Theoretical Physics, Jožef Stefan Institute, P.O. Box 3000, \\ SI-1001 Ljubljana, Slovenia \\ bosiljka.tadic@ijs.si
}

\begin{abstract}
We introduce four algorithms for packet transport in complex networks. These algorithms use deterministic rules which depend, in different ways, on the degree of the node, the number of packets posted down each edge, the mean delivery time of packets sent down each edge to each destination and the time since an edge last transmitted a packet. On scale-free networks all our algorithms are considerably more efficient and can handle a larger load than the random walk algorithm. We consider in detail various attributes of our algorithms, for instance we show that an algorithm that bases its decisions on the mean delivery time jams unless it incorporates information about the degree of the destination node.
\end{abstract}

\section{Introduction}

Complex networks can be used to model a wide range of physical and technological systems. One of the most interesting dynamical problems on network is transport, which can give us some insight into the transport of information in technology based communication networks like the internet [1, the World Wide Web [2, 3] or phone call networks [4. Here we use the term transport to mean transport of particles, which are packets in a network. Thus our model falls within the Network Layer of the OSI Reference Model and the algorithms described in section 3 are routing algorithms that belong to the Network Layer of the OSI Reference Model. Of particular interest is the phenomenon of load in a network, as a function of the rate of packet creation $\mathrm{R}$, which has been investigated for models of communication networks [5, 6, ,7, 8 , and in real networks [9].

Typically the problem of transport is investigated using either a random walk algorithm [6], or the shortest path algorithm used by most internet protocols. The difficulty with these approaches is that random walk algorithm is very inefficient for transport in technology based communication networks and shortest path algorithm requires, for its implementation, information about all connections in network. In this paper we focus on algorithms that use local information about the topology, along with information about the flux of packets between neighbors, the link load and the time taken to deliver packets. We propose four algorithms that use some or all of these properties to deliver packets in a network. 
In section 2 we describe the algorithm that we use to perform numerical simulations of our models. In section 3 we discuss the algorithms that packets use to find their destinations and in section 4 we show our results. In section 5 we summarise our results.

\section{The Program}

A program was written to simulate packet transport on a network that does not depend on the size of the network or its topology. At the beginning of the program an external file with the adjacency matrix of the network is read in. We focus on the internet and consequently we treat nodes in our network as if they were routers. The connections between the routers have the same capacity for all networks. Such a model can not only be used to model internet packet transport but also for a range of transport networks in which the nodes have local routing information.

\section{Each Node}

- Generates a new packet with probability $r=R / N$ and with a randomly chosen destination, where $R$ is a fixed rate for the whole network, and $N$ is the number of nodes in network.

- Stores packets in a queue, which has maximum length is $L=1000$. Packets are despatched from the queue in a first in first out (FIFO) order.

- Sends packets to its neighbours.

\section{Each Node Has Information About}

- The address of all its neighbours (they have unique indices $j$ ).

- The degree of its neighbours - $k(i)$.

- Flow through all its neighbours, which is measured by

- The number of packets posted down each edge to neighbour $i$ - the Link Load - $C(i)$.

- The number of packets sends through neighbour $i$, which have reached their destination - $N_{P}(i)$.

- The sum of the delivery times of all the packets sent through neighbour $i$ that have reached their destination $-T_{P}(i)$.

- The time interval since an edge last transmitted a packet to neighbour $i$ and current time step - $\Delta T(i)$.

The index $i$ enumerates each neighbour of node $k$ and each node keeps all the statistics about its neighbours. Quantities $C(i), N_{P}(i), T_{P}(i)$ and $\Delta T(i)$ describe node $i$ from the perspective of node $k$. Each node is described by its neighbours and all properties can be different for all neighbours that describe node $i$.

The initialization part of the program sets up the network topology, the nodes and all the tables used by them. Inside the main loop a time step is incremented, and within that a loop over all nodes calculates and updates the statistics. The loop over all nodes includes three basics routines, which are run for each node; 
generating new packets, checking its queue for packets with its address and sending packets to its neighbours. Each node generates a packet with a randomly chosen destination with probability $R / N$. The node checks its own queue for packets addressed to itself. When it finds one of these it deletes it from the queue and updates the statistics $N_{P}(i)$ and $T_{P}(i)$ for all the nodes on the packet's path. Each packet keeps track of its own path. The node sends packets to its neighbours by taking the first packet in its queue and checking the packet destination address. If the packet is addressed to one of its neighbour, the node will send it to the neighbour. If it is not, the node will use the algorithm to find where to send the packet. During this posting step the $C(i)$ property is updated. When node $k$ sends packets to node $i$, the number of sent packets $C(i)$ increases. After this loop over all the nodes is completed the quantities $\Delta T(i)$ and the mean delivery time of packets sent down each edge $N_{P}(i) / T_{P}(i)$ are updated for all nodes.

\section{Algorithms}

The most important element in transport is the rule that determines the direction in which a packet is sent. A transport network without a rule is a random walk network. We call this rule the algorithm. It describes how nodes deal with packets and should help packets to get to their destination. Not all algorithms help packets to reach destinations, poor algorithms can easily be worse than the random walk algorithm. All algorithms considered in this paper work with deterministic rules.

The shortest time (ST) algorithm is our basic algorithm that uses information about the mean delivery time $T_{P}(i) / N_{P}(i)$ and the time interval between the last packet that came to node $i$ and actual time step. The ST algorithm finds the minimum value

$$
S_{k}=\min \left[\frac{T_{P}(i)}{N_{P}(i)} \frac{1}{\Delta T(i)}\right]_{i=1 \ldots n}
$$

in order to determine which node to send the packet to. The idea of this algorithm is to try and find the minimum travel time for each packet between source and destination. At the start of the simulation $S$ is equal to 0 for all neighbours. Because the update of $T_{P}(i) / N_{P}(i)$ only occurs when a packet arrives at its destination, it can take a number of time steps before $T_{P}(i) / N_{P}(i)$ becomes nonzero. The inclusion of the reciprocal of $\Delta T(i)$ in $S$ ensures that the algorithm does not get into a state where it never sends a packet down certain links which have a large mean delivery time. This state is particularly likely to occur at the start of the simulation. The inclusion of the reciprocal of $\Delta T(i)$ in $S$ also prevents overcrowding when a node finds a node which is clearly better than all its other neighbours. Hence, because of the inclusion of $\Delta T(i)$ more nodes take part in the transport and in this way the large node do not become overcrowded. Because the algorithm with $T_{P}(i) / N_{P}(i)$ is looking for minimum delivery time we call it the shortest time (ST) algorithm. To start this algorithm, and the STD algorithm, which we will introduce shortly, we use the random walk algorithm. We only use 
the deterministic algorithms at a node when all the values of $S$ of its neighbours are greater than 0 . Without this initial random walk procedure both the ST and the STD algorithms would jam almost immediately. The shortest time and degree (STD) algorithm is a modification of the ST algorithm. It uses information about the local topology, the degree. This helps packets avoid the nodes with the largest degree, which are mostly overcrowded. The idea of incorporating information about the degree of nodes in the transport algorithm was discussed in [10] and 11. In these papers models were introduced in which nodes were selected at a rate proportional to a power of their degree. It was found that the most efficient algorithm was one in which the probability of selecting a node of degree $k$ was proportional to $1 / k$ [10] and [11]. The STD algorithm is defined by

$$
S_{k}=\min \left[\frac{T_{P}(i)}{N_{P}(i)} \frac{1}{\Delta T(i)} k(i)\right]_{i=1 \ldots n}
$$

where $k(i)$ is a degree of node $i$ and $k(i)>1$. This last assumption allows the algorithm to avoid dead-end nodes. A node with degree $k=1$ can only receive a packet that is addressed to itself. The STD algorithm uses both temporal properties and also information about the local connectivity. For transport in a scale-free network the most important nodes are those with the largest degree. But because their neighbours send these nodes a large number of packets the queues at these nodes can become overcrowded. Information about the degree helps the algorithm to avoid these nodes, but it does not mean than they are not used.

The connections and degree (CD) algorithm and the connections, degree and shortest time (CDT) algorithm use information about the link load $C(i)$. Because of this the random walk starting procedure used in the ST and STD algorithms is not required for the $\mathrm{CD}$ and $\mathrm{CDT}$ algorithms. The CD algorithm uses only information about the link load and the degree. The CD algorithm is defined by

$$
S_{k}=\min [C(i) k(i)]_{i=1 \ldots n}
$$

where $C(i)$ is a number of packets that node $k$ sends to node $i$.

For this algorithm $S$ equals 0 at the start, but $C(i)$ is updated almost immediately. When node $k$ sends a packet then it automatically increases the value of $C(i)$. There is no need to wait for information from the destination about the delivery time like in the ST and STD algorithms. In this way CD algorithm improves very quickly and the random walk is not needed. The link load, $C(i)$, quantity helps the algorithm to deliver packets and ensures that almost all nodes take part in the transport. The degree quantity helps to prevent the largest nodes from becoming overcrowded. In this algorithm there is no property that can be optimised, unlike in the ST and STD algorithms where the delivery time is optimised.

The CDT algorithm is intermediate between the CD and the ST algorithms. It optimises the delivery time and does not need the random walk starting procedure because it includes a dependance on the link load, $C(i)$. The dependence on degree prevents large nodes becoming overcrowded. For the CDT algorithm, 
the starting procedure is the same as for the CD algorithm except that we set the ratio

$$
\mathcal{R} \equiv \frac{T_{P}(i)}{N_{P}(i)} \frac{1}{\Delta T(i)} ;
$$

equals to 1 at the start to avoid 0 value. This means that we do not need to start off with a random walk algorithm as in the ST and STD algorithms. The CDT algorithm is defined by

$$
S_{k}=\min \left[\frac{T_{P}(i)}{N_{P}(i)} \frac{1}{\Delta T(i)} C(i) k(i)\right]_{i=1 \ldots n} \quad \text { with } k(i)>1 .
$$

We use the learning property to describe behavior of an algorithm in the beginning. By learning we mean the proportion of links whose value of $S$ has changed since $t=0$. The CD and CDT algorithms learn the most quickly. After 5000 time steps they tried $95 \%$ of links. This is because the link load, $C(i)$, changes when a packet is sent down it whereas $T_{P}(i) / N_{P}(i)$, used by the ST and STD algorithms, only changes when a packet sent down it gets to its destination. That is way the ST and STD algorithms need the random walk starting procedure. With this procedure after 5000 time steps $35 \%$ of links were tried. For the ST algorithm without the random walk starting procedure it was $5 \%$. The speed of learning is important because when a network learns slowly, the network only uses a small proportion of its links for transport over a long period of time, which means that the network is easily jammed when a region of the network becomes overcrowded.

\section{Results}

We consider transport on the scale-free network [12] with $N=1000$ nodes and $m=2$ outgoing links per node. This is an example of uncorrelated network with a power-law distribution of incoming links $P(k) \sim k^{-3}$ for large $k$. When $m=2$ the network includes loops and has relatively small number of connections. Our research show that this network jams for lower values of the posting rate than networks with $m=1$ or $m=3$ and higher. In this work we use a posting rate of $R=0.1$. This means that each node creates a packet with probability $R / N$. The number of time steps for all our simulations is 500,000 . We present results for the STD, CD and CDT algorithms. We do not consider the ST algorithm any further because it isn't stable and always jams.

In figure 19a we show the load in the network, the number of packets that are still in the network. All three algorithms are stable (exhibiting stationary flow). We compared the level of load by finding the mean value of the number of packets in the network. The best algorithm with smallest mean value is the STD algorithm. For the CD and CDT the values are almost the same.

The number of packets in network can be treated as a noise in the network. Measuring the power spectrum of the noise shows that there are correlations in the number of packets on the network. For all three algorithms the power 

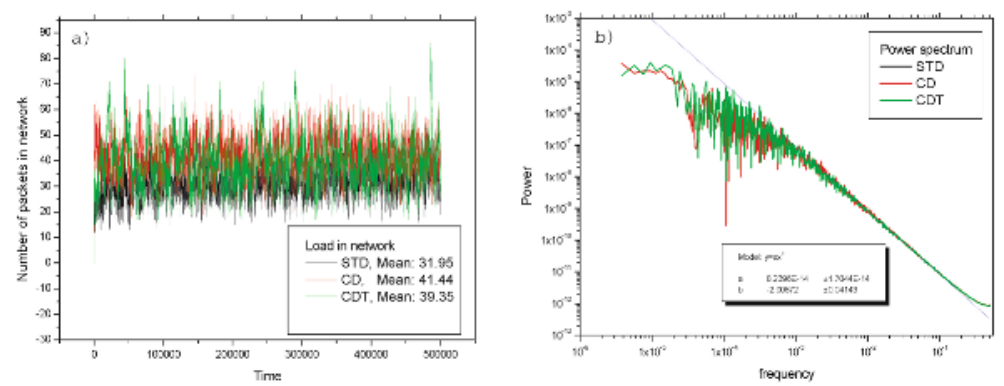

Fig. 1. The load properties. a) Load in the network against time for the STD, CD and CDT algorithms. b) The power spectrum of the load time series.

spectrum (Fig. 10) has the slope $1 / f^{-\phi}$ with $\phi=-2$, indicating short-range correlations.

Further we measured the time interval $\Delta T(i)$, the time that a node waits for next packet. The results for the distribution of $\Delta T(i)$ are shown in figure 2 for all three algorithms. This quantity is important for the improved navigation in the STD and CDT algorithms. We found that without the $\Delta T(i)$ in Eqs ( 2, 5) the networks easily jam.

For the STD algorithm the distribution of $\Delta T(i)$ has a tail and on a double logarithmic scale has a slope $b=-3 / 2$. The cut-off comes from the finite time of the simulation. The first part of the distribution for all algorithms is flat. For the CDT algorithm the function falls faster than for the STD. This is connected with the inclusion of the link load in the CDT algorithm, which means that more links are used and long time intervals of $\Delta T(i)$ do not occur as frequently as in the STD algorithm. The CD algorithm does not make use of $\Delta T(i)$ but we measured it for comparison.

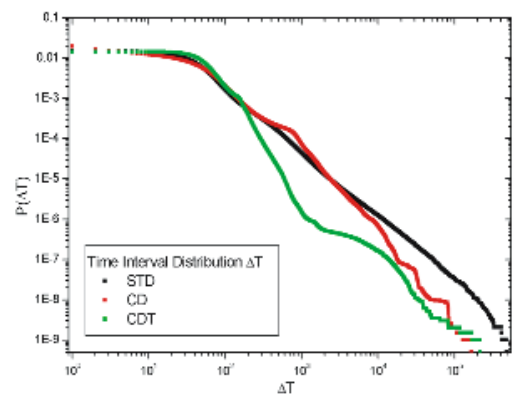

Fig. 2. Distribution of time interval $\Delta T(i)$ for three navigation algorithms

The distribution of packet delivery time $T$ are given in figure 3 a. At long times these distributions appear to be similar for all three algorithms. However the number of packets delivered in a short time differs between the algorithms. 
In particular, for the STD algorithm the probability that a packet is delivered quickly is much higher compared to the CD and CDT algorithms. This is reasonable, because STD algorithm finds the paths with the shortest delivery time. Whereas, the CD and CDT algorithms are distributing the transport across the network making use of the link load $C(i)$ condition. The distribution $P(T)$ for the CDT algorithm interpolated between the STD and CD algorithms reflects its dependence on both the link load $C(i)$ and the shortest time statistics.
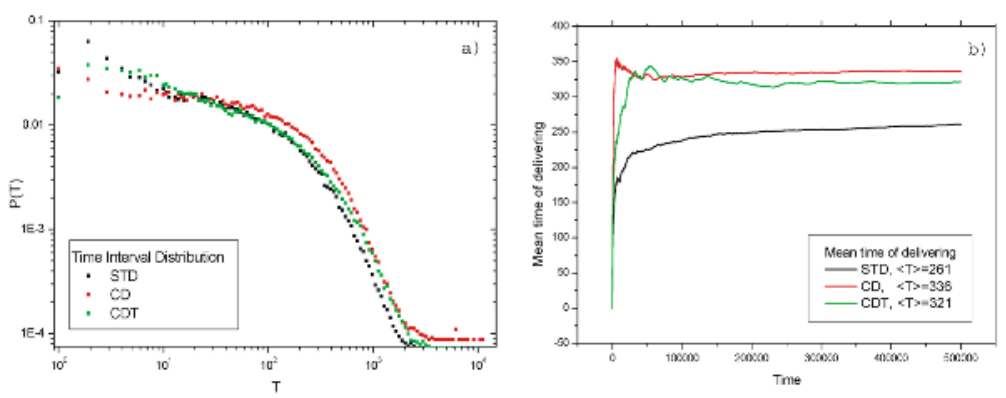

Fig. 3. The time delivery quantities. The distribution of delivery times of packets $(a)$ and the average delivery time $(b)$ for the STD, CD and CDT algorithms.

The time series for the overall mean delivery time of packets (Fig. 3b) show further differences between the navigation algorithms. The algorithms involving the delivery time statistics for $T_{P}(i) / N_{P}(i)$ reach a stable (optimal) level of the average delivery time and maintain it almost constant. On the other hand, the mean delivery time in the CD algorithm increases slowly, suggesting that in this case no optimisation is taking part since none of the involved quantities is requested to be optimized.

The observed result for the STD and CDT algorithms arise through two effects: First, inserting the $\Delta T(i)$ condition in the search rule, nodes intend to send packets through rarely used links. Often these are not the best choices for the transport, however, this mechanisms prevent the network from jamming. Second, including the degree rule in the navigation, nodes preferably send packets to neighbours with a small degree, which potentially makes the delivery times longer on the scale-free network.

\section{Conclusions}

We have introduced several navigation algorithms that are capable to improve the packet transport by optimizing the average delivery times of packets and preventing the network from jamming. We have demonstrated comparatively how these algorithms work in traffic on an uncorrelated scale-free network, which is known to be very prone for jamming [5. These are the algorithms STD and CDT, which are based on the updated statistics of the travel times of packets (global information) and (local) information on the degree of their nearest 
neighbour nodes. For comparison, we have also shown how the traffic behavior changes when parts of these informations are missing, as in the CD and ST algorithms, respectively. In the absence of local rule, the ST navigation leads to traffic jamming relatively quickly, although it is better compared to the random diffusion for the same posting rate. Whereas, in the case of navigation with the CD algorithm (no global information), the traffic seems to be stationary and the results comparable with the other two methods. When the shortest time property is used it needs to be balanced by the degree rule on scale-free networks. The existence of hubs causes traffic congestion for the shortest time algorithm, similarly to the traffic along the topologically shortest paths.

On the technical level, implementation of the STD and CDT algorithms the problem is in finding the accurate value for the edge dependent properties. A node needs a lot of traffic through a given link in order to find its proper time statistics. Because the mean delivery time is very long, it takes a lot of time to set up the edges dependent properties for all nodes. In particular, the algorithms that depend on the time $\Delta T(i)$ and the degree $k(i)$ do not jam but the costs is in learning phase and therefore increased mean delivery time. Using the local property $\Delta T(i)$ in the navigation rule helps in preventing the jamming but at the same time deteriorates the feedback effects from the travel time statistics. The degree property helps the algorithm to avoid nodes with large degree, but it also results in long delivery times. Our results show that in scale free networks we cannot avoid using nodes with large degree.

In the future work the methods developed here can be used for determining the optimal transport path for packets. Some of the related subjects are applications of these algorithms to different or more realistic network geometries, varying posting modes, and increased traffic "bandwidths" at large node.

\section{References}

1. Faloutsos, M., Faloutsos, P. and Faloutsos, C.: Comp. Comm. Rev. 29 (1999) 251

2. Albert, R., Jeong, H. and Barabasi, A.-L.: Nature 401 (1999) 130

3. Huberman, B. and Adamic, L.: Nature 401 (1999) 131

4. Adamic, L. A. , Lukose, R. M., Puniyani, A. R. and Huberman, B. A.: Phys. Rev. E 64 (2001) 046135

5. Tadić, B. and Thurner, S.: Physica A 332 (2004) 566

6. Tadić, B. Thurner, S. and Rodgers, G.J.: Phys. Rev. E 69 (2004) 036102

7. Arenas, A., Diaz-Guilera, A. and Guimera, R.: Phys. Rev. Lett. 86(2001) 3196

8. Sole, R. and Valverde, S.: Physica A 289 (2001) 595

9. Jacobson, V.: in Proceedings of SIGCOMM '88 (ACM, Standford, CA, 1988)

10. Yan, G., Zhuo, T., Hu, B., Fu, Z.-Q. and Wang, B.-H.: cond-mat/0505366 (2005)

11. Yin, C.-Y., Wang, B.-H., Wang, W.-X., Zhou, T. and Yang, H.-J.: condmat/0506204 (2005)

12. Albert, R. and Barabasi, A.-L.: Rev. Mod. Phys. 74 (2002) 47 